\title{
Pre-treatment risk assessment of women with endometrial cancer: differences in outcomes of molecular and clinical classifications in the Slovenian patient cohort
}

\author{
Jure Knez'1,2, Monika Sobocann,2, Urska Belak¹, Rajko Kavalar³, Mateja Zupin ${ }^{4}$, \\ Tomaz Büdefeld ${ }^{4}$, Uros Potocnik ${ }^{4,5}$, Iztok Takac ${ }^{1,2}$

\begin{abstract}
${ }^{1}$ Divison of Gynecology and Perinatology, University Medical Centre Maribor, Maribor, Slovenia
2 Department of Obstetrics and Gynecology, Faculty of Medicine, University of Maribor, Maribor, Slovenia

3 Department of Pathology, University Medical Centre Maribor, Maribor, Slovenia

${ }^{4}$ Centre for Human Molecular Genetics and Pharmacogenomics, Faculty of Medicine, University of Maribor, Maribor, Slovenia

${ }^{5}$ Faculty of Chemistry and Chemical Engineering, University of Maribor, Maribor, Slovenia
\end{abstract}

Radiol Oncol 2022; 56(1): 76-82.

Received 13 May 2021

Accepted 20 August 2021

Correspondence to: Assist. Monika Sobočan, M.D., Division for Gynecology and Perinatology, University Medical Centre Maribor, Ljubljanska ulica 5, SI-2000 Maribor, Slovenia. E-mail: monika.sobocan@gmail.com

Disclosure: No potential conflicts of interest were disclosed.

Background. The aim of this study was to evaluate changes in prognostic risk profiles of women with endometrial cancer by comparing the clinical risk assessment with the integrated molecular risk assessment profiling.

Patients and methods. This prospective study recruited patients with biopsy proven endometrial cancer treated at the University Medical Centre Maribor between January 2020 to February 2021. Patient clinical data was assessed and categorized according to the currently valid European Society of Gynaecological Oncology, European SocieTy for Radiotherapy and Oncology, and European Society of Pathology (ESGO/ESTRO/ESP) guidelines on endometrial cancer. Molecular fumour characterization included determination of exonuclease domain of DNA polymerase-epsilon (POLE) mutational status by Sanger sequencing and imunohistochemical specimen evaluation on the presence of mismatch repair deficiencies (MMRd) and p53 abnormalities (p53abn).

Results. Fourty-five women were included in the study. Twenty-two tumours were of non-specific mutational profile (NSMP) (56.4\%), 13 were classified as MMRd (33.3\%), 3 were classified as p53abn (7.7\%) and 1 was classified as POLE mutated (2.6\%). Six tumours (15.4\%) had multiple molecular classifiers, these were studied separately and were not included in the risk assessment. The clinical risk-assessment classified 21 women (53.8\%) as low-risk, 5 women (12.8\%) as intermediate risk, 2 women as high-intermediate risk (5.1\%), 10 women (25.6\%) as high risk and 1 patient as advanced metastatic (2.6\%). The integrated molecular classification changed risk for 4 women (10.3\%).

Conclusions. Integrated molecular risk improves personalized risk assessment in endometrial cancer and could potentially improve therapeutic precision. Further molecular stratification with biomarkers is especially needed in the NSMP group to improve personalized risk-assessment.

Key words: endometrial cancer; molecular classification; risk assessment

\section{Introduction}

Endometrial cancer is the most common gynaecological malignancy, with an increasing incidence in the developed world. ${ }^{1}$ Most endometrial carcinoma occur in post-menopausal women, however in rare cases they can also affect young women. ${ }^{2}$ In most cases it is diagnosed in early disease stages. With current therapeutic approaches, patients achieve an overall survival (OS) from $74 \%$ to $91 \% .{ }^{1}$ Recently 
published data ${ }^{3}$ on 5-year OS of women with endometrial cancer in Slovenia shows a modest increase in survival between the years $2012-2016(80.6 \%)$ as compared to between the years 1997-2001 (79.8\%). ${ }^{3}$ Endometrial carcinoma are divided, based on their histopathological characteristics, into Type I and Type II carcinoma. Type I carcinomas represent the majority of EC and are of endometrioid subtype. Type II carcinomas are a more hetereogenous group of histopathological subtypes and include clear-cell, serous, mixed histology tumours and carcinosarcomas. ${ }^{4}$

There is a constant need to improve the risk assessment for improved precision in endometrial cancer treatment. An important aim is to identify patients that experience disease reccurrence regardless of the primary early stage endometrial cancer diagnosis. In the last decade, the molecular classification of endometrial cancer has emerged as a feasible possibility to stratify risk in women with endometrial cancer. ${ }^{5}$ The evaluated molecular classification showed, that determining the status of endometrial carcinoma tumours for: i) pathogenic variants of the exonuclease domain of DNA polymerase-epsilon (POLE), ii) mismatch repair deficiency (MMRd) and iii) copy-number high TP53 mutations, enables the determination of specific molecular endometrial cancer subtypes ${ }^{6}$ that could be used in the risk of recurrence assessment. ${ }^{4}$ Clinical trials showed that patients with POLE ultramutated (POLEmut) tumours had a $100 \%$ 10-year recurrence free survival (RFS) versus $80.1 \%$ in POLE wild type (POLEwt) patients. ${ }^{7}$ The recent individual patient meta-analysis has shown that the outcome of POLEmut tumours is good regardless of traditional risk classifiers. ${ }^{8}$ The prognosis is intermediate in MMRd tumours and significantly worse in p53 aberrant tumours. ${ }^{9}$ This led to the incorporation of molecular classification to the recently updated European Society of Gynaecological Oncology, European SocieTy for Radiotherapy and Oncology, and European Society of Pathology (ESGO/ESTRO/ESP) guidelines for endometrial cancer. The current guidelines recommend the use of clinical risk assessment or an integrated clinico-molecular risk assessment, if available. ${ }^{4}$

The molecular risk assessment has been applied for interventions and assessments of adjuvant therapy management decisions. ${ }^{9}$ Based on the current ESGO-ESTRO-ESP guidelines, women with low-risk EC do not need additional adjuvant therapy. For intermediate risk, radiotherapy has been suggested as the optimal course of treatment.
In high-intermediate or some cases of intermediate risk, chemotherapy can be considered in addition to radiotherapy. The guidelines recommend for women with high risk EC to undergo radiotherapy with concurrent chemotherapy. ${ }^{4}$ Abdulfatah et al., reported also the use of molecular classification on biopsy specimens which showed a high level of concordance to hysterectomy specimens and outperformed pre-treatment risk assessment based on histological specimen sample and grade..$^{10}$

Based on the currently available guidelines we aimed to assess the changes that clinical and integrated molecular risk assessment represent in terms of primary patient management.

\section{Patients and methods}

\section{Patients}

This single centre study prospectively recruited consecutive women treated at the University Medical Centre Maribor (UMC Maribor), Slovenia. Participants were recruited from February 2020 to February 2021. Women were eligible to participate in this study if they had a biopsy-proven EC diagnosis and were candidates for surgical treatment.

Informed consent was obtained prior to surgical treatment from all study participants. This study was approved by the Slovenian Ethics Committee for Research in Medicine under the registration number $0120-40 / 2020 / 4$ and was carried out in accordance to the Declaration of Helsinki.

\section{Management plan and risk assessment}

All included women underwent complete diagnostic work-up at our centre, which routinely includes a comprehensive transvaginal ultrasound (TVUS) scan to assess for disease extent. Based on the clinical assessment of myometrial involvement and disease extent, as well as histopathological tumour assessment, a clinical risk prediction was made. Afterwards, they were presented to the interdisciplinary tumour board to plan the optimal treatment. Standard treatment of early stage endometrial cancer is surgical and is most commonly performed by minimally invasive surgery, but open surgical approach is also an option. This depends on the tumour type and patient characteristics. Surgical treatment usually consists of total hysterectomy with bilateral salpingo-oophorectomy. This is most commonly combined with sentinel lymph node biopsy (SLN) or pelvic/paraaortic lymph node dissection (LND). 


\section{Molecular classification of endometrial cancer}

The molecular risk-profile was determined according to current guidelines on endometrial cancer ${ }^{4}$, based on determining the tumour POLE status, the mismatch repair decifiency status (MMRd) of the tumour as well as the p53 tumour expression status. For determining the POLE status of the tumour, DNA was isolated from tissue samples procured from the resected uterus by a pathologist. Tumor DNA was extracted from fresh frozen tissue using a QIAamp DNA Mini Kit (Qiagen $\mathrm{GmbH}$, Hilden, Germany) according to the manufacturer's protocol. DNA purity and concentration were determined using a Synergy ${ }^{\mathrm{TM}} 2$ spectrophotometer (Biotek, Winooski, VT, USA). Primers used for PCR amplification of selected exons 9, 12 and 13 of POLE were designed as described previously. ${ }^{11}$ For amplification of target sequences, we used PCR technique which was performed using DreamTaq DNA Polymerase (Thermo Scientific, Vilnius, Lithuania). Briefly, $2 \mu \mathrm{L}$ of $7 \mathrm{ng} / \mu \mathrm{L}$ DNA was amplified using $8 \mu \mathrm{L}$ mix of $1.0 \mu \mathrm{L}$ DreamTaq buffer, $0.5 \mu \mathrm{L}$ of each primer $(10 \mu \mathrm{M}), 0.2 \mu \mathrm{L}$ dNTPs $(10$ $\mathrm{mM}$ each), $0.05 \mu \mathrm{L}$ DreamTaq DNA polymerase $(5 \mathrm{U} / \mu \mathrm{L})$ and water in a final volume of $10 \mu \mathrm{L}$. Samples were subjected to incubation at $95^{\circ} \mathrm{C}$ for $5 \mathrm{~min}$, then 38 amplification cycles of $95^{\circ} \mathrm{C}$ for 30 sec, $62^{\circ} \mathrm{C}$ for $30 \mathrm{sec}$ and $72^{\circ} \mathrm{C}$ for $30 \mathrm{sec}$, and a final incubation at $72^{\circ} \mathrm{C}$ for $7 \mathrm{~min}$ using The TProfessional Basic Thermocycler (Biometra, Analytik Jena, Jena, Germany). The PCR products were visualised on $2 \%$ agarose gel electrophoresis excised from the gel and purified using a MinElute Gel Extraction Kit (Qiagen $\mathrm{GmbH}$, Hilden, Germany) according to the manufacturer's protocol. Sanger sequencing was performed by Eurofins Genomics (Germany), and nucleotide sequences were manually analysed for the most frequent somatic mutations P286R, V411L, and S459F. ${ }^{12}$

MMRd status was determined by evaluating the immunohistochemical (IHC) markers (MLH1, MSH2, MSH6, PMS2). Previous research showed ${ }^{13}$, that IHC markers represent an appropriate surrogate for screening for MMRd. The p53 protein expression status was evaluated using IHC expression according to current recommendations. ${ }^{14}$

Based on genetic and IHC data, women were grouped, according to the ESGO/ESTRO/ESP guidelines ${ }^{5}$ into the following molecular classification groups: POLE mutated (POLEmut), MMRd, p53 abnormal (p53abn) or non-specific mutational profile (NSMP). If there were multiple molecular classifiers available for a patient, they were analysed separately.

\section{Statistical analysis}

Descriptive statistics were used to evaluate the distribution of each variable. Continuous variables were expressed as mean values with standard deviations. Categorical variables were reported as frequency or percentage. All statistical analyses were performed using SPSS software version 23.0 (IBM Corp., Armonk, NY, USA).

\section{Results}

Molecular characterisation was performed in all 45 women enrolled. Among them, 39 women (86.7\%) were classified according to the current recommendations in one of the four molecular classification groups. Their characteristics are presented in Table 1. Six women (13.3\%) had multiple molecular classifiers. The characteristics of these women are presented in Table 2.

Following the ESGO/ESTRO/ESP guidelines on risk assessment, women were classified according to the clinical risk assessment and the integrated molecular risk (Table 3). Most remained classified as low-risk endometrial cancer, reclassification changed for 4 women.

The risk profiles of three women decreased after integrated molecular risk classification: one was reclassified from high risk to high-intermediate risk and two from intermediate to low-risk. The risk profile of women changed from: i) intermediate to low risk as one woman had a POLEmut tumour, ii) from intermediate to low risk since it was of NSMP and iii) from high to high-intermediate (HIR) risk due to the MMRd classification. In one case, the risk profile increased from low to intermediate risk due to the detected p53abn mutation.

\section{Primary surgical treatment}

Surgical treatment was performed in all patients included in the study. The specifics of the treatment are presented in Table 4 . Thirty women (77\%) underwent a laparoscopic procedure and 9 women $(23 \%)$ an open surgical procedure. The multidisciplinary (MDT) recommendation in cases of pre-operatively persumed low-risk disease is to perform sentinel lymph node biopsy (SLN). If the lymph nodes are not visualized, further LND is generally omitted. In cases of persumed intermediate risk, 
the recommendation in case of SLN visualization failure is to perform LND.

\section{The impact of molecular classification on treatment decisions}

In the post-operative period two women $(5.1 \%)$ died. Two women (5.1\%) rejected the recommended adjuvant therapy. The final analysis was performed for 37 women as depicted in Figure 1.

Our study was observational and the molecular classification did not impact the MDT decision making. Two women which were re-classified from intermediate to low risk by the molecular classification received radiation therapy and one women that has been reclassified from high risk to HIR received radiotherapy and chemotherapy. One patient for whom the risk assesment increased from low to intermediate risk has been recommended no adjuvant treatment. In one patient of the intermediate risk group, the presence of co-morbidities led the MDT to suggest follow-up as the optimal strategy.

\section{Discussion}

In this study we assessed the risk of endometrial cancer for women using the clinical classification and the integrated molecular classification as suggested by the recent ESGO/ESTRO/ESP guide-
TABLE 1. Patient characteristics

\begin{tabular}{|c|c|c|}
\hline \multicolumn{2}{|c|}{ Age at time of diagnosis $(n=39)$} & 65.2 years ( $\min 32-\max 86)$ \\
\hline \multicolumn{2}{|c|}{ Body Mass Index at time of diagnosis $(n=36)$} & $31(17-43)$ \\
\hline \multirow[b]{2}{*}{ Reproductive history } & Parity (median, range) & $2(0-5)$ \\
\hline & $\begin{array}{l}\text { Spontaneuos abortion } \\
\text { (median, range) }\end{array}$ & $0(0-2)$ \\
\hline \multirow{2}{*}{ Menopausal status } & Pre-menopausal & $5(12.8 \%)$ \\
\hline & Post-menopausal & $34(87.2 \%)$ \\
\hline \multirow{2}{*}{ Tumour marker levels } & CA125 $(n=32)$ & $136.3(\min 2-\max 2084)$ \\
\hline & CEA $(n=32)$ & $3.4(\min 2-\max 17)$ \\
\hline \multirow{8}{*}{ FIGO stage $(n=39)$} & IA & $21(54 \%)$ \\
\hline & IB & $8(20.5 \%)$ \\
\hline & $\|$ & $1(2.6 \%)$ \\
\hline & IIIA & $2(5.1 \%)$ \\
\hline & $\| \mathrm{B}$ & $2(5.1 \%)$ \\
\hline & $\mathrm{IIC} 1$ & $3(7.7 \%)$ \\
\hline & $111 \mathrm{C} 2$ & $1(2.6 \%)$ \\
\hline & IV & $1(2.6 \%)$ \\
\hline \multirow{2}{*}{ Tumour type } & Type 1 & $36(92.3 \%)$ \\
\hline & Type 2 & $3(7.7 \%)$ \\
\hline \multirow{4}{*}{$\begin{array}{l}\text { Molecular tumour } \\
\text { classification }\end{array}$} & POLEmut & $1(2.6 \%)$ \\
\hline & MMRd & $13(33.3 \%)$ \\
\hline & NSMP & $22(56.4 \%)$ \\
\hline & p53abn & $3(7.7 \%)$ \\
\hline
\end{tabular}

MMRd = mismatch repair deficient tumour; NSMP = non-specific molecular profile tumour; p53abn = p53 expression abnormal tumour; POLE = DNA polymerase-epsilon; POLEmut $=$ POLE ultramutated tumour

ESGO clinical risk assessment

Integrated molecular risk assessment

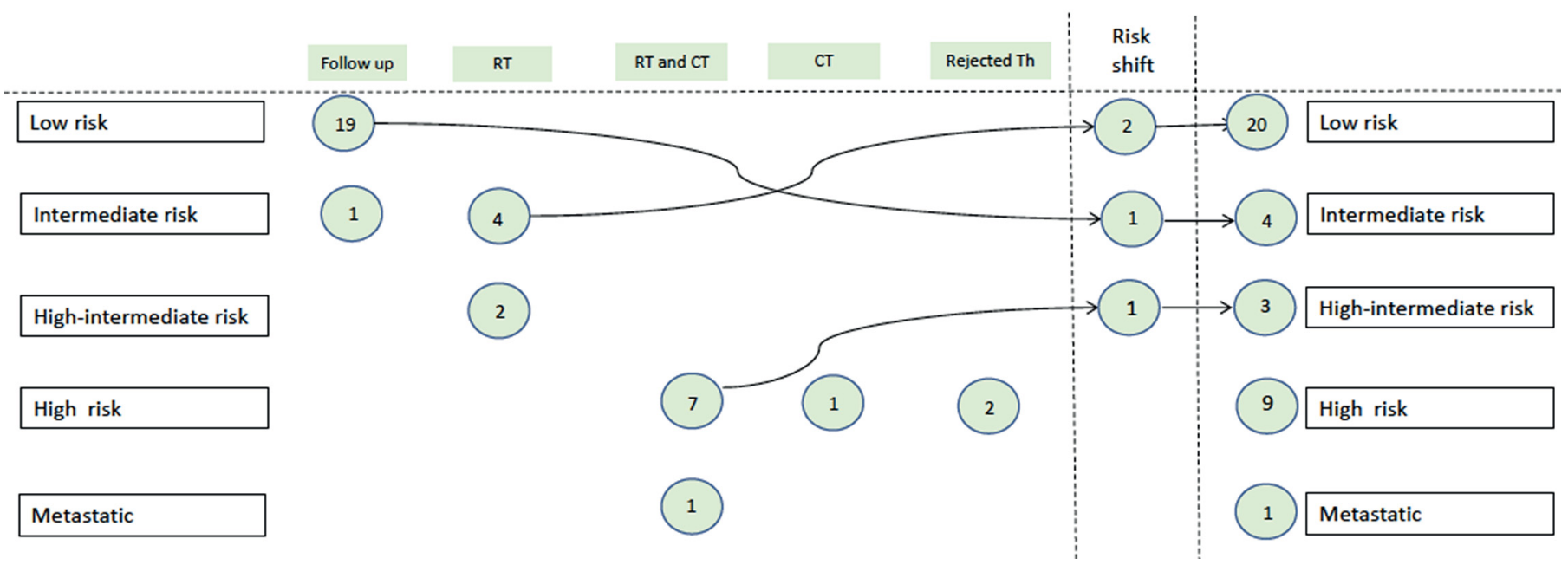

FIGURE 1. Potential impact of risk shifts on adjuvant therapy. Depicted in circles are the absolute numbers of women with endometrial cancer and their adjuvant therapy recommendations. Arrows point to a potential risk shift impacting therapy with the use of the molecular classification.

$\mathrm{CT}=$ chemotherapy; RT = radiotherapy 
TABLE 2. Characteristics of patients with multiple molecular classifiers

\begin{tabular}{|c|c|c|c|c|c|c|c|}
\hline & $\begin{array}{l}\text { Age at time } \\
\text { of diagnosis }\end{array}$ & Multiple-classifier EC & $\begin{array}{l}\text { POLE } \\
\text { variant }\end{array}$ & Tumor type & FIGO stage & $\begin{array}{l}\text { Lymphovascular } \\
\text { invasion }\end{array}$ & $\begin{array}{l}\text { Clinical risk } \\
\text { assessment }\end{array}$ \\
\hline Patient 1 & 76 & POLEmut and p53abn & P286R & endometrioid & IIIC2 & Yes & High \\
\hline Patient 3 & 70 & MMRd and p53abn & wild-type & endometrioid & IA & No & Low \\
\hline Patient 4 & 87 & MMRd and p53abn & wild-type & endometrioid & IIIB & Yes & High \\
\hline
\end{tabular}

MMRd = mismatch repair deficient tumour; p53abn = p53 expression abnormal tumour; POLE = DNA polymerase-epsilon; POLEmut = POLE ultramutated tumour

TABLE 3. Risk assessment

\begin{tabular}{ccc}
\hline & & Number of patients (\%) \\
\hline & Low risk & $21(53.8 \%)$ \\
$\begin{array}{c}\text { ESGO Clinical } \\
\text { Risk Group }\end{array}$ & Intermediate risk & $5(12.8 \%)$ \\
& High-intermediate risk & $2(5.1 \%)$ \\
& High risk & $10(25.6 \%)$ \\
Antegranced metastatic & Low risk & $1(2.6 \%)$ \\
molecular risk & Intermediate risk & $22(56.4 \%)$ \\
& High-intermediate risk & $4(10.3 \%)$ \\
& High risk & $3(7.7 \%)$ \\
\hline
\end{tabular}

ESGO = European Society of Gynaecological Oncology

lines. Out of 45 women enrolled in our study, the risk evaluation through integrated molecular risk groups was possible for 39 women. Six women had multiple molecular classifiers, which precluded further risk assessment using the current molecular classification. In the remaining group, assessment of molecular risk decreased in three and increased in one woman.
The classification of women with endometrial cancer into risk groups based on clinical and molecular data aims to improve individualised treatment according to the tumour biological potential. According to the currently valid guidelines ${ }^{4}$, histopathological characteristics, including lymphvascular space invasion (LVSI) are the cornerstone of risk stratification. This is one of the reasons that shifts in risk groups between clinical and integrated molecular groups were present, as LVSI has been identified as an important marker of prognosis in low-risk endometrial cancer and is associated with adverse outcomes. ${ }^{15}$ In comparison with a study of Oberndorfer et al. ${ }^{16}$ which compared molecular risk with the guidelines on clinical risk assessment in endometrial cancer published in $2016^{17}$, there were less shifts in the clinical risk assessment in our study. Therefore, by using the newly implemented clinical or integrated molecular risk assessment, comparable risk assessment is achieved. Our study is the first to compare the clinical and molecular risk stratification in the cohort of Slovenian patients. The clinical value of this approach needs to be verified in further prospective studies.

The nature of our study was observational and the molecular subtype was not taken into consideration for decision-making in regards to surgical

TABLE 4: Analysis of surgical treatment of women based on ESGO Clinical Risk Group assesment. SLN - sentinel lymph node biopsy, LND - lymphadenectomy

\begin{tabular}{ccccccc}
\hline $\begin{array}{c}\text { ESGO integrated } \\
\text { molecular risk }\end{array}$ & $\begin{array}{c}\text { Total number of } \\
\text { women }\end{array}$ & $\begin{array}{c}\text { Open } \\
\text { surgery }\end{array}$ & Laparoscopic & SLN & LND & $\begin{array}{c}\text { Unilateral SNB and } \\
\text { contralateral LND }\end{array}$ \\
\hline Low risk & $22(56.4 \%)$ & $2(5.1 \%)$ & $20(51.3 \%)$ & $18(46.2 \%)$ & 0 & 0 \\
treatment
\end{tabular}

HIR = high-intermediate risk; LND = lymphadenectomy; SLN = sentinel lymph node biopsy 
or adjuvant therapy. Table 4 represents the surgical approach taken for women based on the pretreatment assessment and the final ESGO Risk Group classification. The body of knowledge on using the integrated molecular classification for decision making on surgical treatment is scarce. Histopathological pre-operative evaluation might not always be concordant with post-operative diagnosis ${ }^{18,19}$ and could potentially impact the MDT to suggest less invasive surgical procedures. This is especially true in recognizing serous EC. In these instances based on the molecular characteristics such as TP53 mutations ${ }^{20}$, integrated molecular evaluation could potentially enable individualized therapy already in the primary surgical setting.

Considering the changes in risk assessment for patients within our study, if using the integrated molecular assessment, two patients would most likely been recommended follow-up instead of radiotherapy, thus de-escalating their therapy based on their individual biological features. One patient would have been, based on her biological characteristics upgraded from low to intermediate risk. Especially in the low-intermediate and intermediate-high risk group, it is important to identify the patients correctly. Without appropriate adjuvant therapy, these patients were found to be at a approximately $30 \%$ higher risk than if adjuvant therapy was offered..$^{21}$ One of our patients was downgraded from high risk to the HIR risk group. While this individual has received radiotherapy and chemotherapy, this is also a valid strategy of treatment in the HIR risk group ${ }^{4}$ and the decision process most likely would have not changed for her. Participants in our study have been mostly classified as NSMP (56.4\%). NSMP tumours are currently classified in the low-risk or intermediate risk ${ }^{4}$ based on a combination with other histopathological characteristics. As NSMP represents the largest group of currently classified tumours this shows the need for further refinements of molecular risk stratification. ${ }^{22,23}$ A potential additional marker which has shown potential prognostic value, especially in the intermediate risk groups, is L1CAM expression ${ }^{23,24}$ and in low risk endometrial cancer also mutation of CTNNB1. ${ }^{25}$ Further evaluation of these and other novel markers could enable us to further individualise and stratify risk assessment in this large and clinically diverse group. ${ }^{26}$

An important consideration in our study is how to evaluate and incorporate risk assessment for women with multiple classifiers. There were six women with multiple classifiers in which four tumours were p53abn and POLEmut. Multiple classi- fiers MMRd and p53abn were present in two women. Recently, Leon-Castilo et al. ${ }^{27}$ published data on their cohort of patients with multiple-classifier endometrial cancer. They supported the bioinformatic clustering of TCGA data which stated that MMRd-p53abn tumours mostly clustered around MMRd tumours and p53-POLEmut tumours mostly clustered around POLEmut tumours. Clinical outcomes showed that patients with MMRdp53abn had a 5-year recurrence free survival (RFS) of $92.2 \%$ and patients with POLEmut-p53abn endometrial cancer a 5 -year RFS of $94.1 \% .{ }^{27}$ This also supports the hypothesis that these tumours are biologically less aggressive than single classifier p53abn tumors, which had a 5-year RFS of $48 \% .{ }^{28}$ Further research and biomarker development is therefore needed to evaluate the appropriate approaches for patient treatment in cases of non-specific mutational profiles and multiple-classifiers.

The strength of our study is that it is the first prospectively designed study to evaluate the implementation of molecular risk stratification to endometrial cancer patients in Slovenia. The limitation is the low number of patients included. The followup data is not yet available and the clinical implications of this approach are yet to be determined.

\section{Conclusions}

The introduction of molecular risk stratification in the management of women with endometrial cancer represents a significant shift from the established clinical practice. Several adjustments to the routine workflow and significant additional resources are necessary in order to implement this approach to the clinics. Our data shows that in comparison to the current clinical risk stratification based on clinical and histopathological data, this may lead to change in management in a small proportion of women. The clinical value of this remains to be proven in further prospective studies. It is also important to note that the molecular risk stratification is not applicable to all women and refinements of the current classification with additional biomarkers are likely to improve and further de-escalate treatment in certain subtypes of endometrial cancer in the future.

\section{Acknowledgement}

The project was funded by the Institutional Research funding of UMC Maribor, reg. no IRP- 
2019/02-13 and by the Slovenian Research Agency (research core funding P3-0067).

\section{References}

1. Morice P, Leary A, Creutzberg C, Abu-Rustum N, Darai E. Endometrial cancer Lancet 2016; 387:1094-108. doi: 10.1016/S0140-6736(15)00130-0

2. Repše-Fokter A. Endometrial cancer in young woman. Acta MedicoBiotechnica 2020; 13: 60-4.

3. Zadnik V, Zagar T, Lokar K, Tomsic S, Konjevic AD, Zakotnik B. Trends in population-based cancer survival in Slovenia. Radiol Oncol 2021; 55: 42-9. doi: 10.2478/raon-2021-0003

4. Concin N, Matias-guiu X, Vergote I, Cibula D, Mirza MR, Marnitz S, et al. ESGO / ESTRO / ESP Guidelines for the management of patients with endometrial carcinoma. Int J Gynecol Cancer 2021; 31: 12-39. doi: 10.1136/ ijgc-2020-002230

5. Urick ME, Bell DW. Clinical actionability of molecular targets in endometria cancer. Nat Rev Cancer 2019; 19: 510-21. doi: 10.1038/s41568-019-0177-x

6. Cancer Genome Atlas Research Network, Kandoth C, Schultz N, et al. Integrated genomic characterization of endometrial carcinoma. Nature 2013; 497: 67-73. doi: 10.1038/nature12113

7. Wortman BG, Creutzberg CL, Putter H, Jürgenliemk-Schulz IM, Jobsen JJ, Lutgens LCHW, et al. Ten-year results of the PORTEC-2 trial for high-intermediate risk endometrial carcinoma: improving patient selection for adjuvan therapy. Br J Cancer 2018; 119: 1067-74. doi: 10.1038/s41416-018-0310-8

8. McAlpine JN, Chiu DS, Nout RA, Church DN, Schmidt P, Lam S, et al Evaluation of treatment effects in patients with endometrial cancer and POLE mutations: An individual patient data meta-analysis. Cancer 2021; 26 5400-10. doi: 10.1002/cncr.33516

9. Vermij L, Smit V, Nout R, Bosse T. Incorporation of molecular characteristics into endometrial cancer management. Histopathology 2020; 76: 52-63. doi: 10.1111/his.14015

10. Abdulfatah E, Wakeling E, Sakr S, Al-Obaidy K, Bandyopadhyay S, Morris R, et al. Molecular classification of endometrial carcinoma applied to endometrial biopsy specimens: Towards early personalized patient management. Gynecol Oncol 2019; 154: 467-74. doi: 10.1016/j.ygyno.2019.06.012

11. Malentacchi F, Turrini I, Sorbi F, Projetto E, Castiglione F, Vergoni F, et al Identification of a gene panel for endometrioid endometrial cancer: a possible prognostic value? Reprod Sci 2020; 27: 592-8. doi: 10.1007/s43032 019-00059-8

12. McAlpine J, Leon-Castillo A, Bosse T. The rise of a novel classification system for endometrial carcinoma; integration of molecular subclasses. J Patho 2018; 244: 538-49. doi: 10.1002/path.5034

13. Raffone A, Travaglino A, Cerbone M, Guida M, Insabato L, Zannoni GF, et al. Diagnostic accuracy of immunohistochemistry for mismatch repair protein as surrogate of microsatellite instability molecular testing in endometrial cancer. Pathol Oncol Res 2020; 26: 1417-27. doi: 10.1007/s12253-02000811-5

14. Köbel M, Ronnett BM, Singh N, Soslow RA, Gilks CB, McCluggage WG. Interpretation of P53 immunohistochemistry in endometrial carcinomas. Int J Gynecol Pathol 2019; 38: S123-S131. doi: 10.1097/PGP.0000000000000488

15. Ayhan A, Şahin H, Sari ME, Yalçin I, Haberal A, Meydanli MM. Prognostic significance of lymphovascular space invasion in low-risk endometrial cancer. Int J Gynecol Cancer 2019; 29: 505-12. doi: 10.1136/ijgc-2018-000069

16. Oberndorfer F, Moling S, Hagelkruys LA, Grimm C, Polterauer S, Sturdza A, et al. Risk reclassification of patients with endometrial cancer based on tumor molecular profiling: First real world data. J Pers Med 2021; 11: 1-11. doi: 10.3390/jpm11010048

17. Colombo N, Creutzberg C, Amant F, Cibula D, Mirza MR, Marnitz S, et al. ESMO-ESGO-ESTRO consensus conference on endometrial cancer. Int $J$ Gynecol Cancer 2016; 26: 2-30. doi: 10.1097/igc.0000000000000609

18. Han G, Sidhu D, Duggan MA, Arseneau J, Cesari M, Clement PB, et al. Reproducibility of histological cell type in high-grade endometrial carcinoma. Mod Pathol 2013; 26: 1594-604. doi: 10.1038/modpathol.2013.102
19. Sobočan M, Ogrizek AM, Ledinek T, Takač I, Knez J, Importance of preoperative ultrasound examination and pathological tumour evaluation in the management of women with endometrial cancer. Eur J Obstet Gynecol Reprod Biol 2021; 257: 121-126. doi: 10.1016/j.ejogrb.2020.12.029

20. Bogani G, Ray-Coquard I, Concin N, Ngoi NYL, Morice P, Enomoto T, et al. Uterine serous carcinoma. Gynecol Oncol 2021; 162: 226-34. doi: 10.1016/j. ygyno.2021.04.029

21. Van Den Heerik ASVM, Horeweg N, De Boer SM, Bosse T, Creutzberg CL. Adjuvant therapy for endometrial cancer in the era of molecular classification: radiotherapy, chemoradiation and novel targets for therapy. Int Gynecol Cancer 2021; 31: 594-604. doi: 10.1136/ijgc-2020-001822

22. Talhouk A, McConechy MK, Leung S, Li-Chang HH, Kwon JS, Melnyk N, et al. A clinically applicable molecular-based classification for endometrial cancers. Br J Cancer 2015; 113: 299-310. doi: 10.1038/bjc.2015.190

23. Kommoss FKF, Karnezis AN, Kommoss F, Talhouk A, Taran FA, Staebler A, et al. L1cam further stratifies endometrial carcinoma patients with no specific molecular risk profile. Br J Cancer 2018; 119: 480-6. doi: 10.1038/s41416018-0187-6

24. Weinberger V, Bednarikova $M$, Hausnerova J, Ovesna $P$, Vinklerova $P$, Mina $L$, et al. A novel approach to preoperative risk stratification in endometrial cancer: The added value of immunohistochemical markers. Front Oncol 2019; 9: 1-13. doi: 10.3389/fonc.2019.00265

25. Imboden S, Tapia C, Scheiwiller $\mathrm{N}$, Kocbek $\mathrm{V}$, Altermatt $\mathrm{HJ}$, Janzen J et al. Early-stage endometrial cancer, CTNNB1 mutations, and the relation between lymphovascular space invasion and recurrence. Acta Obstet Gynecol Scand 2020; 99: 196-203. doi: 10.1111/aogs.13740

26. Kolehmainen A, Pasanen A, Tuomi T, Koivisto-Korander R, Butzow R, Loukovaara M. Clinical factors as prognostic variables among molecular subgroups of endometrial cancer. PLoS One 2020; 15:1-12. doi: 10.1371/ journal.pone.0242733

27. León-Castillo A, Gilvazquez E, Nout R, Smit VT, McAlpine JN, McConechy M, et al. Clinicopathological and molecular characterisation of 'multiple-classifier' endometrial carcinomas. J Pathol 2020; 250: 312-22. doi: 10.1002/ path.5373

28. Leon-Castillo A, De Boer SM, Powell ME, Mileshkin LR, Mackay HJ, Leary A et al. Molecular classification of the PORTEC-3 trial for high-risk endometrial cancer: Impact on prognosis and benefit from adjuvant therapy. J Clin Oncol 2020; 38: 3388-97. doi: 10.1200/JCO.20.00549 